\title{
Simultaneous Aortic Valve Replacement with Nephrectomy: A Case Report
}

\author{
Ajmer Singh ${ }^{1}$ Aashish Jain ${ }^{1}$ Yatin Mehta ${ }^{2}$ Naresh Trehan ${ }^{3}$ \\ ${ }^{1}$ Division of Cardiac Anaesthesia, Medanta-The Medicity, Gurugram, \\ Haryana, India \\ 2 Institute of Critical Care and Anesthesiology, Medanta-The \\ Medicity, Gurugram, Haryana, India \\ ${ }^{3}$ Division of Cardiac Surgery, Medanta Heart Institute, Medanta-The \\ Medicity, Gurugram, Haryana, India \\ J Card Crit Care TSS 2018;2:98-101
}

\begin{abstract}
Address for correspondence Ajmer Singh, MD, Division of Cardiac Anaesthesia, Institute of Critical Care and Anesthesiology, Medanta-The Medicity, Sector-38, Gurugram 122001, Haryana, India (e-mail: ajmersingh@yahoo.com).
\end{abstract}
Abstract
Keywords
- aortic stenosis
- aortic valve
replacement
- nephrectomy
- simultaneous surgery
- critical stenosis
- hypertension

A 60-year-old man presented to the authors' institution and underwent simultaneous surgery for severe aortic stenosis and left renal mass. Potential benefits of a combined cardiac and noncardiac procedure are discussed. The literature, however, is scarce on standards of management of patients with two coexisting life-threatening conditions.

\section{Introduction}

Diseases of the cardiovascular system and neoplastic disorders are common causes of death worldwide. Risk of sudden death in patients with asymptomatic severe aortic stenosis (AS) is less common than in symptomatic patients, but it remains a concern. ${ }^{1}$ Delayed excision of neoplastic tumor, after performing cardiac surgery, can potentially lead to spread of cancerous cells by immunosuppression caused by cardiopulmonary bypass (CPB). ${ }^{2}$ The decision to perform simultaneous surgeries was taken to achieve a long-lasting remission.

\section{Case Report}

A 60-year-old, obese (height $179 \mathrm{~cm}$, weight $126 \mathrm{~kg}$, body mass index $39.3 \mathrm{~kg} / \mathrm{m}^{2}$, body surface area $2.41 \mathrm{~m}^{2}$ ), hypertensive man presented to the hospital for general medical checkup. Physical examination revealed a midsystolic ejection murmur heard best at right upper sternal border radiating to the neck. Subsequent cardiac evaluation showed a bicuspid, calcific aortic valve with severe AS (aortic valve area $0.9 \mathrm{~cm}^{2}$, peak/mean transvalvular gradients
$95 / 62 \mathrm{~mm} \mathrm{Hg}$ ), and left ventricular (LV) ejection fraction of $45 \%$ on echocardiography. The dimensions of aorta were: annulus $24 \mathrm{~mm}$, aortic root $28 \mathrm{~mm}$, and ascending aorta $35 \mathrm{~mm}$. Routine laboratory parameters and X-ray chest were normal. Ultrasound scan of the abdomen revealed a large left renal mass measuring $8.4 \times 7.7 \mathrm{~cm}$. Computed tomography (CT) of the abdomen confirmed ultrasound findings ( - Fig. 1). Whole-body positron emission tomography (PET) CT scan showed absence of thrombus in the inferior vena cava or right atrium; it also ruled out metastasis of the tumor. After consultations with cardiothoracic surgical and urology teams, a simultaneous aortic valve replacement (AVR) and left open radical nephrectomy were planned.

In the preoperative holding area, before induction of anesthesia, a lumbar epidural catheter (L3-4 interspace) was placed using loss of resistance to saline method. A test dose of $3 \mathrm{~mL}$ of $2 \%$ lignocaine was administered. Under standard cardiac monitoring including pulmonary artery (PA) catheter, anesthesia was induced with midazolam, fentanyl, and etomidate. Anesthesia was maintained with intermittent doses of midazolam, fentanyl, atracurium, and oxygen in air with isoflurane. A multiplane transesophageal echocardiography (TEE) probe was inserted, which
DOI https://doi.org/ 10.1055/s-0039-1685132 ISSN 2457-0206.
Copyright @2018 Official Publication of The Simulation Society (TSS), accredited by International Society of Cardiovascular Ultrasound (ISCU).

\section{License terms}

(ㅇ) (1) $\Theta \circledast$ 


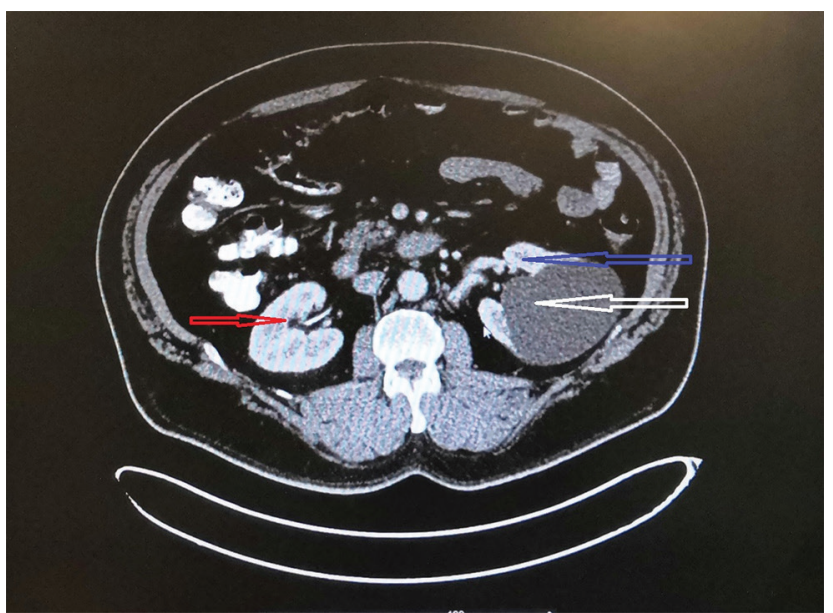

Fig. 1 Computed tomography scan of abdomen showing right kidney (red arrow), left kidney (blue arrow), and left renal mass (white arrow).

confirmed preoperative findings. A mid-sternotomy approach was used for AVR (bioprosthetic Epic valve $23 \mathrm{~mm}$ ), and CPB management consisted of crystalloid prime, moderate hypothermia, flow rate of $2.4 \mathrm{~L} / \mathrm{min} / \mathrm{m}^{2}$, mean arterial pressure $>65 \mathrm{~mm} \mathrm{Hg}$, hematocrit $\geq 24 \%$, and cold cardioplegic arrest. The aortic cross-clamp and СРB times were 62 and 85 minutes, respectively. Post-CPB TEE evaluation revealed normal functioning of bioprosthetic aortic valve, with mean transvalvular gradient of $12 \mathrm{~mm} \mathrm{Hg}$. No inotropes were required during weaning from CPB or thereafter. After achieving hemostasis, one pleural and two mediastinal drains were inserted and sternum was closed. For intraoperative analgesia, $20 \mathrm{~mL}$ of bupivacaine $0.5 \%$ was administered. The urology team made left subcostal incision, approximately 4 to $5 \mathrm{~cm}$ away from the sternotomy incision ( - Fig. 2 ), and opened the abdomen in layers. After mobilization of colon and Gerota's fascia, left kidney along with cystic mass on its upper pole was identified. Left ureter, renal artery, and renal vein were clipped, followed by en masse removal of left kidney and mass (-Fig. 3). Wound was closed in layers after putting in a drain. The total duration of surgery was 410 minutes, and an estimated blood loss of approximately $600 \mathrm{~mL}$ was replaced with transfusion of 1 unit of packed red cells. Intraoperative urine output was $700 \mathrm{~mL}$. After 12 hours of mechanical ventilation, trachea was extubated and the patient was mobilized on day 2. Postoperative pain was managed with buprenorphine $(0.15 \mathrm{mg}$ once a day) administered epidurally for 72 hours, followed by parenteral analgesics. The visual analog score (VAS) was kept below 4. Intensive care unit (ICU) and hospital stays were 3 and 8 days, respectively. Histopathology of the specimen revealed clear-cell renal cell carcinoma grade pT2aNO. A follow-up at 3 months revealed no complication related to either surgical procedure.

\section{Discussion}

This case highlights the importance of regular physical checkup and thorough evaluation of all organ systems even in asymptomatic patients. A delay in detection and

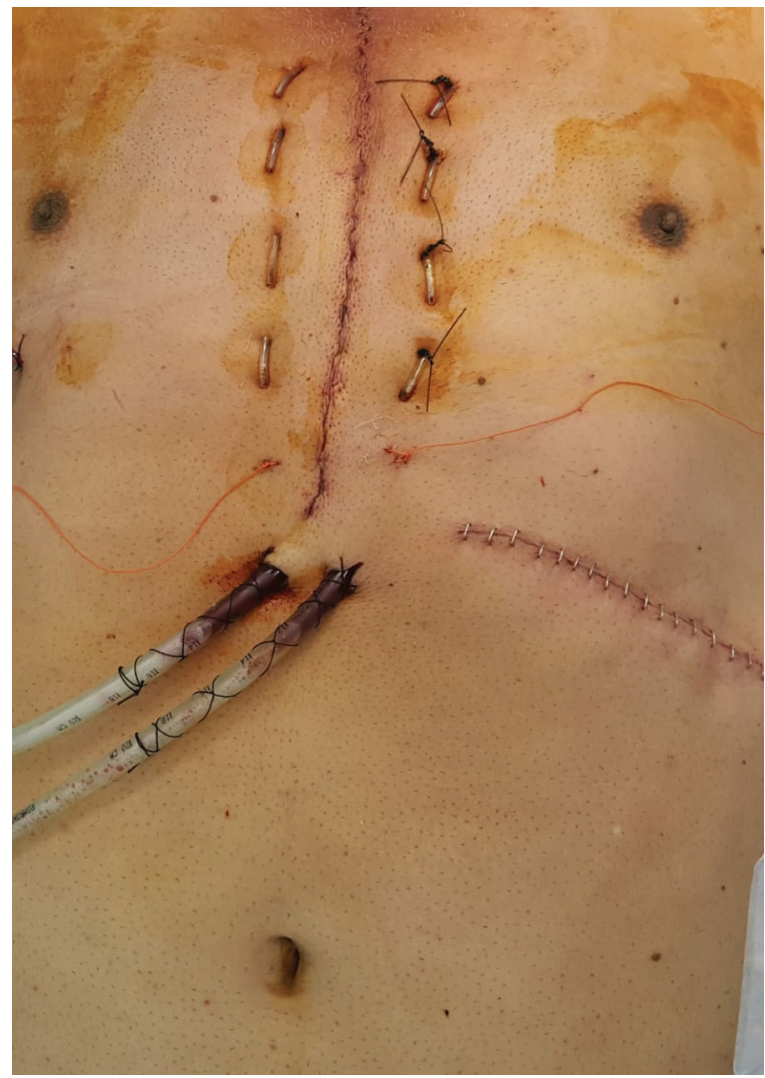

Fig. 2 Skin incisions of chest and abdomen.

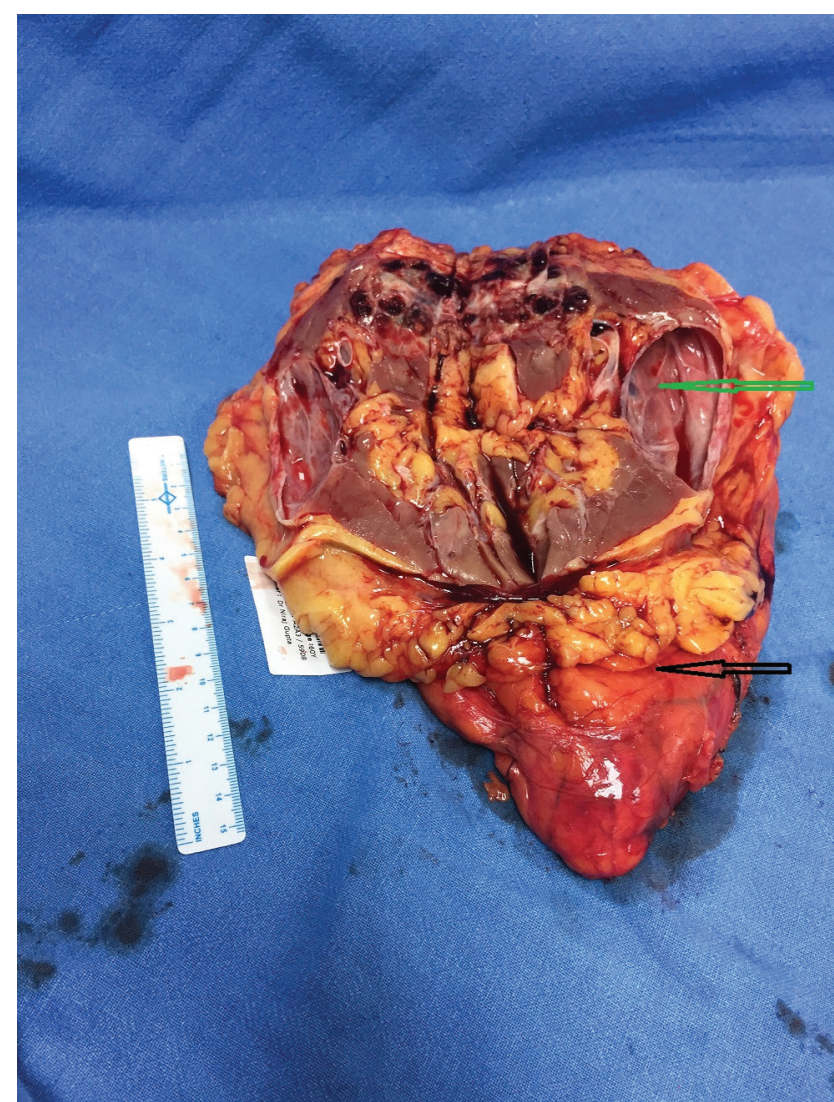

Fig. 3 Surgical specimen showing cystic left renal mass (green arrow) and left kidney (black arrow). 
management of severe AS and renal tumor in this case could have resulted in sudden cardiac death or metastasis of cancerous cells to other organs.

Patients with severe AS can be managed in one of the following ways: AVR, transcatheter aortic valve implantation (TAVI), or balloon aortic valvuloplasty (BAV). BAV is reserved for very high-risk patients, with noncalcific AS, severe heart failure, and/or cardiogenic shock, often as a bridge to definitive therapy. ${ }^{3}$ TAVI is the preferred option for patients with high ( $\geq 4 \%$ ) EuroSCORE, presence of severe comorbidities, previous cardiac surgery, age $\geq 75$ years, frailty, and restricted mobility. Surgical AVR, however, remains the gold standard for treatment of AS. Cardiac evaluation in asymptomatic patients can lead to timely detection of cardiac disease(s) and can help avoid cardiovascular complications.

It is customary to stage the cardiac and noncardiac procedures. We, however, chose a concomitant approach. The decision to perform cardiac surgery followed by abdominal surgery in the same sitting was taken after discussion with both operating teams. Cardiac surgery, considered "clean surgery," was performed first to avoid the possible risks of mediastinitis and/or infection of bioprosthetic valve. Second, hemodynamic perturbations associated with major abdominal surgery can have adverse consequences in the presence of severe AS. The largest published series of simultaneous (15 cases) and staged (14 cases) surgery for renal tumors and concomitant cardiac diseases has always performed cardiac surgery first to reduce the risk of cardiac complications. ${ }^{4}$ In addition, performing AVR first and postponing renal tumor excision may have put the patient at risk of immunosuppressive effects of CPB. This approach could have resulted in deleterious effects on tumor spread and growth, besides increased cost of a staged surgery. There is substantial evidence to support the harmful effects of CPB contributing to dissemination of malignancy. ${ }^{5}$ A good longterm result of two patients undergoing simultaneous renal tumor excision and AVR has been described previously. ${ }^{6}$ We did not adopt the extended sternotomy technique (mid-sternotomy incision extended to subcostal incision) described by Budrikis et al, owing to the risk of spread of infection from abdominal cavity to chest cavity. ${ }^{7}$

Renal protective measures taken during perioperative period included maintenance of euvolemia, avoidance of hemodilution, maintenance of hemodynamics (mean arterial pressure $\geq 65 \mathrm{~mm} \mathrm{Hg}$ ), maintenance of adequate flow and perfusion during $\mathrm{CPB}$, limiting the duration of $\mathrm{CPB}$, and avoidance of nephrotoxic agents (e.g., aminoglycosides, angiotensin-converting enzyme inhibitors, angiotensin receptor blockers, nonsteroidal anti-inflammatory agents, and contrast agents). Renal near-infrared spectroscopy monitoring would have probably been helpful for prediction of acute kidney injury caused by ischemia, but most studies related to this modality have been done on children. It is possible to identify renal vessel and measure renal blood flow by the use of TEE imaging in approximately 60\% of adult cases, but it is still a controversial technique with low feasibility and steady learning curve. ${ }^{8}$
Patients undergoing AVR and major abdominal surgery are at risk of developing low cardiac output in the postoperative period. Common causes of low cardiac output following AVR include hypovolemia, myocardial depression (preexisting LV systolic dysfunction, inadequate myocardial protection, ischemia-reperfusion injury, myocardial stunning, prolonged CPB duration, diastolic dysfunction), LV outflow obstruction producing systolic anterior motion, valve dysfunction, and arrhythmias (loss of atrial contribution in atrial fibrillation, bradycardia, heart block).9,10 Patients with low cardiac output are at higher risk of developing infectious, neurologic, respiratory, renal, or gastrointestinal complications. Blood loss during tumor excision was anticipated, and we had enough monitoring tools (PA catheter, TEE) to diagnose hypovolemia and other causes of low cardiac output.

Postoperative pain management is an important issue in an obese patient undergoing simultaneous thoracic and abdominal incisions. The risk of developing postoperative pulmonary dysfunction and hypoxemia was very high in this patient, due to presence of obesity, use of general anesthesia consisting of narcotics and inhalational agents, thoracic and upper abdominal incisions, impaired diaphragmatic function, etc. Use of lumbar epidural analgesia probably helped the patient by allowing early mobilization and by better preservation of pulmonary functions, in addition to its other known potential benefits. ${ }^{11}$ We followed the standard ASRA (American Society of Regional Anesthesia and Pain Medicine) recommendations of inserting epidural catheter 60 minutes before heparinization and preparedness to postpone surgery in the event of a bloody tap.

In conclusion, the combined surgical approach for treatment of severe AS and renal mass was successful and resulted in good outcome. An anesthesiologist, as a member of a multispecialty team, plays a major role in the decision making, resource utilization, and perioperative care of a combined cardiac and noncardiac procedure.

\section{Conflict of Interest}

None.

\section{References}

1 Lund O, Nielsen TT, Emmertsen K, et al. Mortality and worsening of prognostic profile during waiting time for valve replacement in aortic stenosis. Thorac Cardiovasc Surg 1996;44(6):289-295

2 Hill GE, Whitten CW, Landers DF. The influence of cardiopulmonary bypass on cytokines and cell-cell communication. J Cardiothorac Vasc Anesth 1997;11(3):367-375

3 Eltchaninoff $\mathrm{H}$, Durand $\mathrm{E}$, Borz $\mathrm{B}$, et al. Balloon aortic valvuloplasty in the era of transcatheter aortic valve replacement: acute and long-term outcomes. Am Heart J 2014;167(2):235-240

4 Andrushchuk U, Ostrovsky Y, Krasny S, et al. Simultaneous or staged surgery in patients with kidney tumors and concomitant cardiac disease. Cent European J Urol 2017;70(4):356-361

5 Fu Q, Li QZ, Liang DG, Ruan XH, Wang ZX, Wei MX. Early and long-term results of combined cardiac surgery and neoplastic resection in patients with concomitant severe heart disease and neoplasms. Chin Med J (Engl) 2011;124(13):1939-1942 
6 Litmathe J, Atmaca N, Menghesha D, Krian A. Combined procedures using the extracorporeal circulation and urologic tumor operation experiences in six cases. Interact Cardiovasc Thorac Surg 2004;3(1):132-135

7 Budrikis A, Jievaltas M, Al Assaad S, Kinduris S. Simultaneous nephrectomy and coronary artery bypass grafting through extended sternotomy. J Cardiothorac Surg 2012;7:79-82

8 Zhu D, Yu H, Zhou Y, et al. Feasibility of measuring renal blood flow using transesophageal echocardiography in pediatric patients undergoing cardiac surgery. J Cardiothorac Vasc Anesth 2012;26(1):39-45
9 Lomivorotov VV, Efremov SM, Kirov MY, Fominskiy EV, Karaskov AM. Low- cardiac-output syndrome after cardiac surgery. J Cardiothorac Vasc Anesth 2017;31(1):291-308

10 Pérez Vela JL, Jiménez Rivera JJ, Alcalá Llorente MA, et al; en representación del Grupo ESBAGA. Low cardiac output syndrome in the postoperative period of cardiac surgery. Profile, differences in clinical course and prognosis. The ESBAGA study. Med Intensiva 2018;42(3):159-167

11 Liu S, Carpenter RL, Neal JM. Epidural anesthesia and analgesia. Their role in postoperative outcome. Anesthesiology 1995;82(6):1474-1506 